\title{
Proprotein convertase 1 mediated proneuropeptide proteolytic processing in ischemic neuron injury
}

\author{
Tang $\mathrm{SS}^{2}$, Liang $\mathrm{ZY}^{1}$, Guo $\mathrm{LR}^{2}$, Zhang $\mathrm{JH}^{3}$, Zhou $\mathrm{D}^{1}$ \\ Department of Neurosurgery, Guangdong General Hospital, Guangdong Academy of Medical Sciences, \\ 106 Zhongshan Er Road, Guangzhou City, Guangdong Province, China. zhoudong5413@163.com
}

\begin{abstract}
BACKGROUND: Pro-protein processing mechanism plays an important role in neuron injury.

OBJECTIVE: To study the protein convertase 1 (PC1) mediated processing mechanism, the ischemic cellular or tissue proPC1/PC1 or proCgA/CgA (pro-chromogranin A) was analyzed.

METHODS: NS20Y differentiated cells were stressed by $0-6 \mathrm{~h}$ of oxygen and glucose deprivation (OGD) in glucosefree DMEM and an anaerobic jar environment. Ischemic C57BL/J mouse model was established by performing 60-min of middle cerebral artery occlusion (MCAO) operation and subsequent 4 or 24 -h reperfusion. The TUNEL, immunochemistry, and Western blot methods were used to detect protein expression in ischemic cells or tissues. RESULTS: The OGD or MCAO stress caused substantial cell death in a dose-dependent manner $(p<0.05$ or $0.01)$. With the increasing OGD dose, proPC1 and PC1 proteins gradually increased $(p<0.05$ or 0.01$)$ whereas proCgA and $\mathrm{CgA}$ proteins decreased $(p<0.05)$. In vivo the proPC1 and PC1 expressions presented with a peak at $4-h$ and then decreased at $24-h$ reperfusion $(p<0.05$ or 0.01$)$. The tissue proCgA and CgA proteins decreased with the increasing reperfusion time $(p<0.05)$.

CONCLUSIONS: The results suggest that the increasing PC1 expression promoted the transformation of proCgA into CgA or smaller peptides, i.e. Pancreastatin or Secretoneurin, and the PC1 mediated processing plays a critical role (Fig. 4, Ref. 15). Text in PDF www.elis.sk.

KEY WORDS: pro-protein processing, neuron ischemic injury, pro-protein convertase 1, chromogranin A.
\end{abstract}

\section{Introduction}

The profiles of neuropeptides in functional tissue may determine their responses to ischemic stress, apoptosis, or necrosis. The PCs mediated proneuropeptide processing and maturation plays an important role in neuron stress and injury (1-4). PC1 is a calciumdependent endoprotease expressed in neural and endocrine cells (5) and involved in the biosyntheses of many neuropeptides such as $\mathrm{CgA}$ and pancreastatin (PST) et al $(6,7)$. It was reported that kainic acid increases PC1 expression in neural cells (8). Marcinkiewicz $\mathrm{M}$ et al (9) reported that PC1 was induced in the transected sciatic nerve and was present in cultured Schwann cells or the transected peripheral nerve injury. Zhang JH et al (10) reported that proPC1

${ }^{1}$ Department of Neurosurgery, Guangdong General Hospital, Guangdong Academy of Medical Sciences, Guangzhou, China, ${ }^{2}$ Department of Biochemistry and Molecular Biology, School of Basic Courses, Guangdong Pharmaceutical University, Guangzhou, China, and ${ }^{3}$ Department of Obstetrics \& Gynecology, Guangdong Provincial Corps Hospital, Guangzhou Medical University, Guangzhou, China

Address for correspondence: Dong Zhou, PhD, MD, Department of Neurosurgery, Guangdong General Hospital, Guangdong Academy of Medical Sciences, 106 Zhongshan Er Road, Guangzhou city, Guangdong Province, China. Zipcode 510080.

Phone: +86.20 .83827812 ext. 61524

Acknowledgement: This research was supported by Natural Grant of Guangdong Provincial (S2012010009953, 2014A030313589, and 2015A030313843).
$(87 \mathrm{kDa})$ automatically activates into mature PC1 $(81 \mathrm{kDa})$ in adequate conditions, such as $5.0 \mathrm{pH}$ and higher $\mathrm{Ca}^{2+}$.

Chromogranin A (CgA), one member of acidic secretory protein family, is expressed in all neuroendocrine tissues. Some reports demonstrated that proCgA is a substrate of $\mathrm{PC} 1$ which transforms proCgA (about $60 \mathrm{kDa}$ ) into $\mathrm{CgA}(50 \mathrm{kDa})$. proCgA or $\mathrm{CgA}$ can be further processed into smaller neuropeptides such as Pancreastatin or Secretoneurin $(11,12)$.

In the paper, $\mathrm{PC} 1$ mediated proCgA processing in ischemic neurons was observed in vitro and in vivo. Our long- term goal is to understand PC molecular mechanisms in some diseases, which will help the diagnosis and treatment of the diseases.

\section{Materials and methods}

\section{Animals}

C57BL/J mice (20-25 g, Rat No: 4400850000219) were purchased from Guangdong Experimental Animal Center (China). All experiments were performed in accordance with the Institutional Guidelines for the Care and Use of Laboratory Animals. The animals were acclimated to a light-dark cycle of $12: 12 \mathrm{~h}$ by housing them in individual cages at a temperature of $26 \pm 1{ }^{\circ} \mathrm{C}$. The studies were approved by the Animal Centers on animal care. After all mice used were raised for 2 days, they were randomly grouped.

\section{Focal cerebral ischemic model}

30 of C57 mice (half male and half female) were randomly 
609-612

allocated into three groups (sham, 4-, and 24-h MCAO groups, n $=10$ ). In 20 out of the C57 mice the left middle cerebral artery occlusion models (MCAO) were performed with a modified method of Rousselet $\mathrm{E}$ et al (13), and in 10 of the $\mathrm{C} 57$ mice sham operations were performed. Briefly, the mice were anesthetized with 4 $\%$ chloral hydrate and the MCAO operation was performed by inserting a nylon suture (Beijing Sunbio Biotech Co., China) into the lumen of internal carotid artery through carotid artery. After 60-min MCAO, the suture was withdrawn to allow 4 or 24-h reperfusion. The seven mouse cortices in each group were dissected. According to TTC staining (2, 3, 5-triphenyltetrazolium chloride) (Shanghai Yuanye Biotechnology Co., Ltd. China), the tissues from the ischemic cortical regions of MCAO territory were taken and kept at $-80{ }^{\circ} \mathrm{C}$ for Western blot and ELISA assay $(\mathrm{n}=$ 7). The remaining three cortices in each group were fixed in $4 \%$ of paraformaldehyde solution and $8 \mu \mathrm{m}$-thick frozen coronal sections were stained with immunofluorescent method.

\section{Oxygen and glucose deprivation model}

Oxygen and glucose deprivation (OGD) models were prepared as previously described (14). The NS20Y cells were differentiated with $10^{-4} \mathrm{M}$ ctp-cAMP (Sigma Chemical Co, USA) for $24 \mathrm{~h}$ and incubated in glucose-free DMEM (Invitrogen Incorporation, USA) and in an anaerobic jar (AnaeroPack system, Japan) for 0-6 h OGD treatment.

\section{Western blotting analysis}

The ischemic mouse or NS20Y cells were homogenized with a buffer consisting of $50 \mathrm{mmol} / \mathrm{L}$ Tris-HCL (pH 7.5), $1 \%$ Triton $\mathrm{X}-100,10 \%$ glycerol, and protease inhibitors, respectively. Protein concentration was quantified by a Bradford method. $50 \mu \mathrm{g}$ of protein were fractionated by SDS-PAGE (12\%) and blotted onto a PVDF membrane (Millipore Co., USA), then incubated with a rabbit polyclonal antibody against PC1 (1:2000 diluted, Abcam Co., USA) or against CgA (1:500 diluted, Biowordle Co, USA,), following with enzyme-catalyzed chemiluminescence (ECL) method.

\section{Immunofluorescent analysis}

For cellular immunofluorescent staining, the dishes with OGD cells were washed two times in PBS for 10 min, fixed in $4 \%$ paraformaldehyde-PBS buffered for $15 \mathrm{~min}$, and subsequently washed one time with PBS for $5 \mathrm{~min}$. The dishes or mouse brain sections were incubated in $4 \%$ goat serum for $30 \mathrm{~min}$, then incubated with primary antibody (PC1 1:500 or CgA 1:200) at $4{ }^{\circ} \mathrm{C}$ overnight. After washed 3 times with PBS, they were incubated in Cy3-conjugated secondary antibody. Lastly, DAPI staining was performed. The fluorescent signals were examined and photographed with a Leica epifluorescence microscope.

\section{Death rate analysis}

Brain ischemic stroke was analyzed in the TTC staining (15). TTC staining allows a precise delineation of lesioned and nonlesioned brain areas for subsequent dissection of selected tissue pieces for molecular analysis.
The OGD cell death was analyzed in TUNEL staining (Roche Diagnostics, USA) using a commercial kit and cell counting method. The TUNEL staining was referred to the company protocol. 10 pictures with nuclei (DAPI) or positive cells each section were randomly taken in a fluorescent microscope, respectively. In the experiment three sections $(n=3)$ were used at one timepoint. The death rates were obtained by using the average ratio of the positive cell numbers / DAPI nucleus numbers and $X^{2}$ test in statistics.

\section{Results}

Evaluation of ischemic injury in vitro and in vivo

The TTC staining reveals white areas as infracted regions of cerebral cortex and striatum (Fig. 1A). The injury region in the 24hour group shows significantly higher sizecompared to the sham or 4-hour group (data not shown). The OGDs caused substantial NS20Y cell death in a dose-dependent manner ( $p<0.05$ or 0.01 ) (Figs 1B1 and 1B2).

\section{Expression of proPC1 and PC1 after ischemic injury}

Western blotting results indicated that the expression levels of proPC1 in the 4-h reperfusion group significantly increased compared to that in the sham group $(\mathrm{p}<0.05)$ or in the $24-\mathrm{h}$ reperfusion group $(\mathrm{p}<0.01)$ (Fig. 1C1). Although PC1 expression increased at 4-h reperfusion, it was not statistically different from the sham group. The PC1 expression significantly decreased after 24-h reperfusion $(\mathrm{p}<0.05)$ (Fig. 1C2). For the OGD cells, the proPC1 or $\mathrm{PC} 1$ protein levels increased with OGD in a dose-dependent manners ( $\mathrm{p}<0.05$ or 0.01$)$ (Figs 1D1 and 1D2).

The results of the cortex tissue immunofluorescent staining showed that PC1 is expressed in neuron cell body or dendrite. The PC1-positive neurons in the sham group were significantly decreased compared to those in the 4-h reperfusion group $(\mathrm{p}<$ $0.01)$ or the $24-\mathrm{h}$ reperfusion group $(\mathrm{p}<0.05)$ (Figs $2 \mathrm{~A} 1$ and $2 \mathrm{~A} 2)$. The $\mathrm{PC} 1$ expression in the ischemic cells significantly increased in a OGD dose-dependent manner $(\mathrm{P}<0.05)$ (Figs 2B1 and 2B2).

\section{Expression of proCgA and $\mathrm{CgA}$ protein after ischemic injury}

Western blotting method confirmed the proCgA or CgA expression in neurons. The proCgA or $\mathrm{CgA}$ protein expression decreased with the reperfusion time (in vivo) (Fig. 3A) or in OGD dose-dependent manner (in vitro) $(p<0.05)$ (Fig. 3B).

The result of $\mathrm{CgA}$ immunofluorescent staining in the 4-h reperfusion or the 24-h reperfusion group was significantly decreased

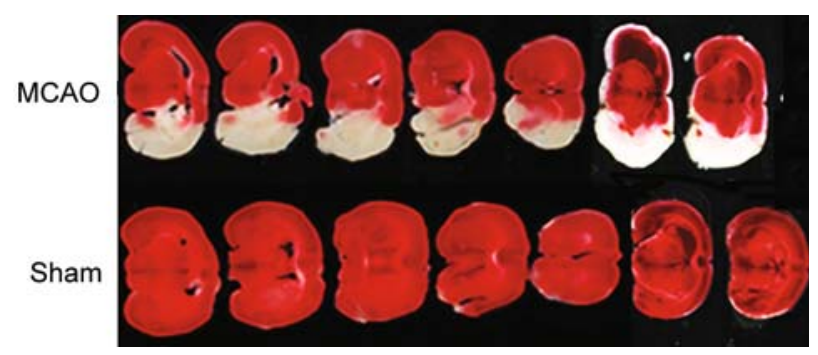

Fig. 1. The cortical infracted area in TTC staining $(n=7)$. 


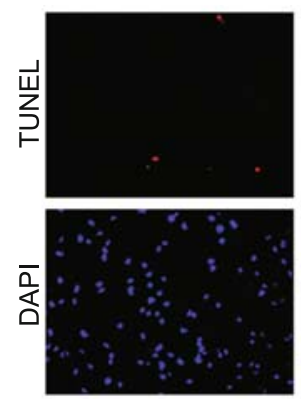

(A) OGD Oh
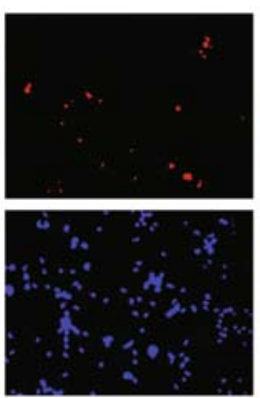

$2 \mathrm{~h}$

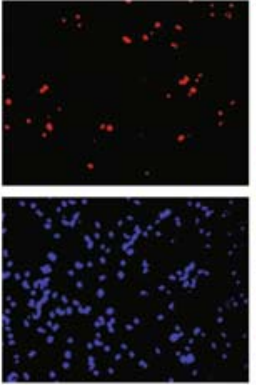

$4 \mathrm{~h}$

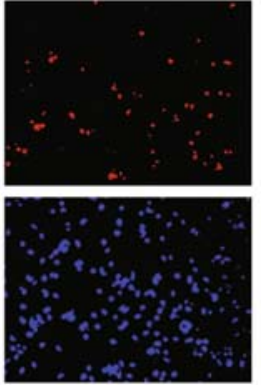

$6 \mathrm{~h}$

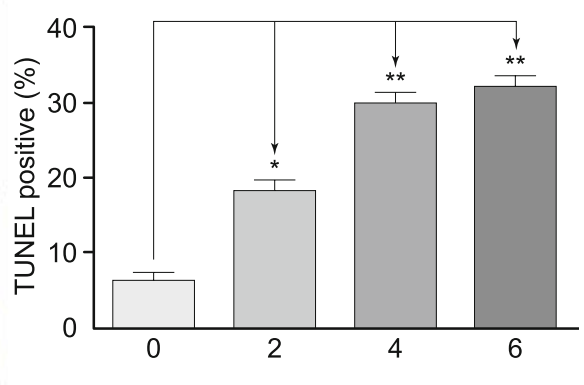

(B)

OGD time (h)

Fig. 2. Analysis of death rate of OGD NS20Y cells $(n=3)$. A: TUNEL staining pictures; B: TUNEL staining analysis $(* p<0.05$ or 0.01 vs the 0-h OGD group).

\section{A (in vivo)}

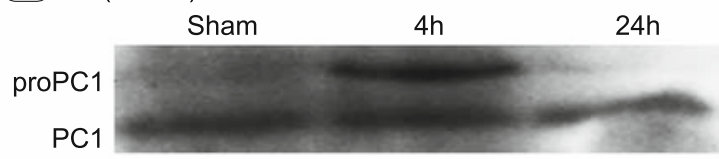

$\beta$-Actin
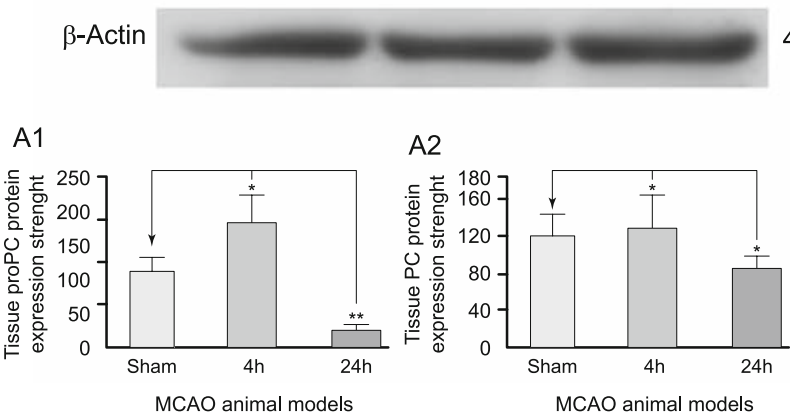

B (in vitro)

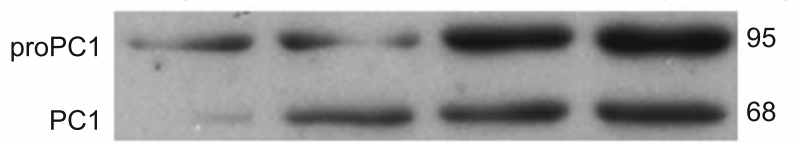

42

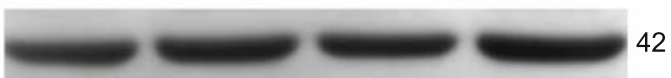

B1

B2

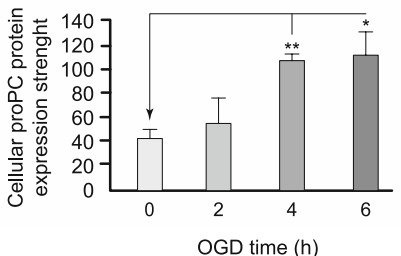

Fig. 3. Western blot analysis of mouse cortical (A) or ischemic cells (B) proPC1 or PC1 protein (n= 7 or 3). A1, A2, B1, B2: Statistical analysis, $* \mathrm{p}<0.05$ or 0.01 vs the sham or the OGD 0-h group.

A
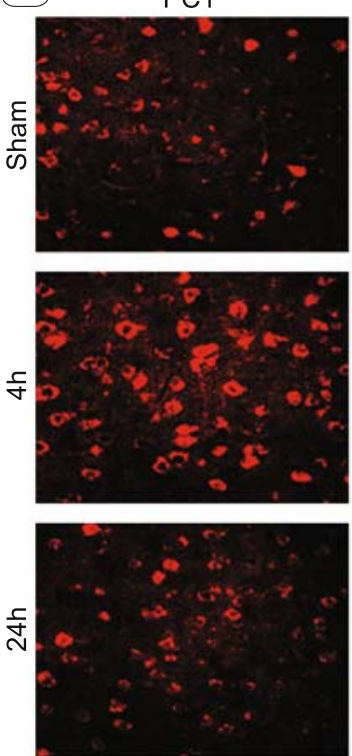

DAPI
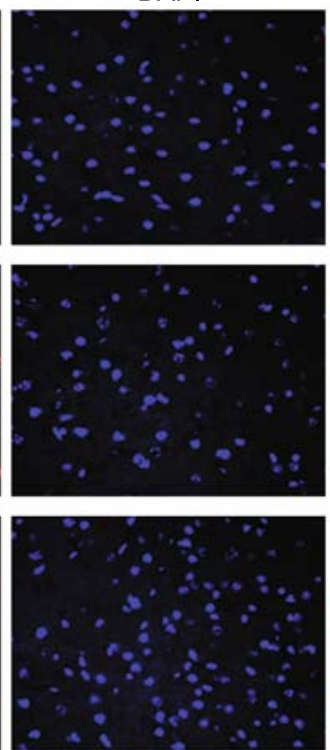

Merge

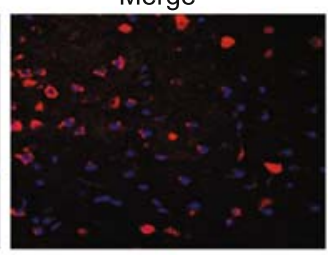

B
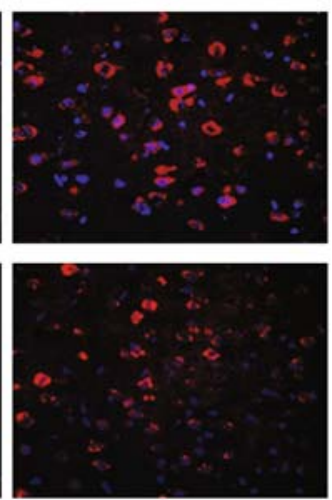

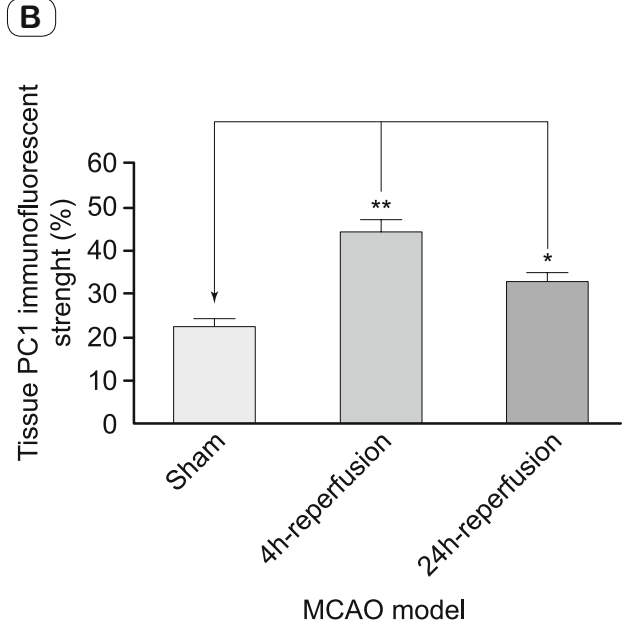

Fig. 4. Immunofluorescent analysis of PC1 (proPC1) in mouse cortex frozen sections $(\mathrm{n}=3)$ ). A: PC1 (proPC1) immunofluorescent staining (PC1 positive: red color; DAPI: blue color; scale bar $50 \mu \mathrm{m}$, mag.10*10). B: PC1 (proPC1) expression analysis in mouse cortex sections. * $\mathrm{p}<$ 0.05 or 0.01 vs the sham group. 
609-612

compared to that in the sham group $(\mathrm{p}<0.05)$ (Fig. 4A). Although the results of cellular CgA immunofluorescent staining show a decreasing trend with OGD time, there is no significant difference between the different OGDs groups (Fig. 4B).

\section{Discussion}

In brain cells, the production of active neuropeptides relies on the presence and proper function of a set of neuropeptide processing enzymes including PC1. Some publications reported that proprotein processing, i.e. PC2 and CPE mediated processing is involved in ischemic injury of neurons. Researchers believe that these alterations of proprotein processing system determine the death or survival of neural cells in some pathological process such as ischemic stress.

The death rates of brain cortical cells or the OGD-inducing NS20Y cells were obtained by using TTC or TUNEL staining. The injury area in the 4-h or 24-h reperfusion group showed to be statistically significantly bigger compared to the sham group. The OGD caused substantial NS20Y cell death in a dose-dependent manner, suggesting that cell death rates were positively correlated with reperfusion time or OGD time. The expression level of proPC1 or PC1 in the 4-h reperfusion group significantly increased compared to that in the sham group or the 24-h reperfusion group, indicating that the peak compensation of proPC or PC expression occurred at 4-h reperfusion. Their expressions significantly decreased after 24 -h reperfusion, suggesting that the in vivo proPC 1 or $\mathrm{PC} 1$ expressions became weaker with increasing death rate. For the OGD cells, proPC1 or PC1 protein levels increased with OGD in a dose-dependent manners, whereas with the increasing ischemic dose, the expression of proCgA or $\mathrm{CgA}$ protein decreased, which suggests that the increasing $\mathrm{PC} 1$ promotes the $\mathrm{CgA}$ production or further degradation.

The results of the $\mathrm{CgA}$ immunofluorescent staining in the 4-h reperfusion or the 24-h reperfusion group significantly decreases compared to that in the sham group. Although the results of the cellular CgA immunofluorescent staining show a decreasing trend, there is no significant difference between the different OGDs, which shows different results with the in vivo experiment. The Western blot results coincide with the IHC ones.

The in vitro and vivo experimental results suggest that with increasing stress, proPC1 was aggregated and transformed into more $\mathrm{PC}$, which induced the corresponding substrate proCgA into $\mathrm{CgA}$ or further smaller molecules. The tissue protein expressions show inconsistent with the cellular ones, which suggests that the in vivo experiment was regulated by whole body system, whereas the $2-6$ h cellular OGDs may not reach the peak stress. Many protein expressions show obvious decrease after a peak OGD due to the death of most cell.

The in vivo proPC1 aggregation gradually disappeared with increasing reperfusion time. The gradual increase of proPC1 may be to compensate for the insufficiency of the active PC1. This reveals that PC1 may be very important for rescue ischemic injury.

After OGD, the exhaustion of ATP is the first event. The maturation, moving and secretion of the vesicle containing $\mathrm{PC} 1$ and its substrates need a lot of ATP. In a word, the cascades of OGD $\rightarrow$ ATP block $\rightarrow$ vesicle maturation block may lead to inhibition of transformation of proCgA into $\mathrm{CgA}$.
In conclusion, the proPC1 aggregation and the $\mathrm{CgA}$ decrease adversely exacerbated the neuronal cell injury. This study reveals a new facet of the biological effect of $\mathrm{PC} 1$ processing following ischemia-reperfusion injury.

\section{References}

1. Zhan S, Zhou A, Piper C, Yang T. Dynamic changes in proprotein convertase 2 activity in cortical neurons after ischemia/reperfusion and oxygenglucose deprivation. Neural Regen Res 2013; 8 (1): 83-89.

2. Tang SS, Zhang JH, Liu HX, Zhou D, Qi R. Pro-protein convertase-2/ carboxypeptidase-E mediated neuropeptide processing of RGC-5 cell after in vitro ischemia. Neurosci Bull 2009; 25 (1): 7-14.

3. Zhou A, Minami M, Zhu X, Bae S, Minthorne J, Lan J, Xiong ZG et al. Altered biosynthesis of neuropeptide processing enzyme carboxypeptidase E after brain ischemia: molecular mechanism and implication. J Cereb Blood Flow Metab 2004; 24 (6): 612-622.

4. Noel F, Gumin GJ, Raju U, Tofilon PJ. Increased expression of prohormone convertase-2 in the irradiated rat brain. FASEB J 1998; 12 (15): 1725-1730.

5. Seidah NG, Chretien M. Pro-protein convertases of subtilisin/kexin family. Methods Enzymol 1994; 175-188.

6. Udupi V, Lee HM, Kurosky A, Greeley GH Jr. Prohormone convertase-1 is essential for conversion of chromogranin A to pancreastatin. Regul Pept 1999; 83 (2-3): 123-127.

7. Eskeland NL1, Zhou A, Dinh TQ, Wu H, Parmer RJ. Chromogranin A processing and secretion: specific role of endogenous and exogenous prohormone convertases in the regulated secretory pathway. J Clin Invest 1996; 98 (1): 148-156.

8. Meyer A, Chrétien P, Massicotte G, Sargent C, Chrétien M, Marcinkiewicz M. Kainic acid increases the expression of the prohormone convertases furin and PC1 in the mouse hippocampus. Brain res 1996; 732 (1-2): 121-132.

9. Marcinkiewicz M, Savaria D, Marcinkiewicz J. The pro-protein convertase $\mathrm{PC} 1$ is induced in the transected sciatic nerve and is present in cultured Schwann cells: comparison with PC5, furin and PC7, implication in pro-BDNF processing. Brain Res Mol Brain Res 1998; 59 (2): 229-246.

10. Zhang JH, Zhou D, You J, Tang BS, Li PY, Tang SS. Differential processing of neuropeptide proprotein in human breast adenocarcinoma. $\mathrm{J}$ Endocrinol Invest 2013; 36 (9): 745-752.

11. Zhou A, Bloomquist BT, Mains RE. The prohormone convertases PC1 and PC2 mediate distinct endoprotcolytic cleavages in a strict temporal order during proopiomelanocortin hiosynthctic processing. J Biol Chem 1993; 268 (3): 1763-1769.

12. Shyu WC, Lin SZ, Chiang MF, Chen DC, Su CY, Wang HJ, Liu RS, et al. Secretoneurin promotes neuroprotection and neuronal plasticity via the Jak2/Stat3 pathway in murine models of stroke. J Clin Invest 2008; 118 (1): 133-148.

13. Rousselet E, Kriz J, Seidah NG. Mouse model of intraluminal MCAO: cerebral infarct evaluation by cresyl violet staining. J Vis Exp 2012; 6 (69): 1-5.

14. Chi W, Meng F, Li Y, Li P, Wang G, Cheng H, Han S, et al. Impact of microRNA-134 on neural cell survival against ischemic injury in primary cultured neuronal cells and mouse brain with ischemic stroke by targeting HSPA12B. Brain Res 2014; 1592: 22-33.

15. Kramer M, Dang J, Baertling F, Denecke B, Clarner T, Kirsch C, Beyer C, et al. TTC staining of damaged brain areas after MCA occlusion in the rat does not constrict quantitative gene and protein analyses. J Neurosci Methods 2010; 187 (1): 84-89.

Received May 7, 2017. Accepted May 30, 2017. 